Miriam Abu Sharkh

\title{
Warum ratifizieren Länder internationale
}

\section{Kinderarbeitskonventionen?}

\author{
Eine Ereignisanalyse der Ratifizierung des „Übereinkommens über das \\ Mindestalter für die Zulassung zur Beschäftigung، \\ (ILO-Konvention Nr. 138)
}

\begin{abstract}
Der Beitrag untersucht anband einer Ereignisanalyse von 1973 bis 2005, weshalb Länder die Konvention Nr. 138 der Internationalen Arbeitsorganisation ratifizieren, die Kinderarbeit untersagt. Die Ratifizierung internationaler Konventionen ist bedeutsam, da sie eine Anerkennung weltgesellschaftlicher Normen darstellt. Die Konvention gegen Kinderarbeit ist besonders interessant, weil sie sebr konkrete Anforderungen an die Länder stellt, ibre Arbeitsmarktgesetze und -strukturen zu verändern und somit sozioökonomische Kosten nach sich zieben kann. Weltgesellschaftliche Argumente des Neoinstitutionalismus der Stanforder Schule bestätigend, wird quantitativ-empirisch gezeigt, dass die Ratifizierung wenig von ökonomischen Merkmalen eines L andes oder dessen außenpolitischem Kalkül geprägt wird, wie es die Entwicklungsliteratur und die realpolitische Perspektive der Internationalen Beziehungen nabe legen. Vielmebr prägen globale Diskurse und Initiativen, die durch weltweit agierende Organisationen in die Länder getragen werden, nationalstaatliches Ratifizierungsverbalten nacbbaltig. Wesentlich bierfür sind die organisatoriscbe Einbindung eines Staates in die Weltgesellschaft und weltgesellschaftliche Ereignisse. Es zu folgern, dass UN-Organisationen und Konferenzen sowie NGO-Aktivitäten nicht als "talk shops" abzutun sind, sondern die Anerkennung weltgesellschaftlicher Normen durch Staaten erbeblich vorantreiben können.
\end{abstract}

Kinderarbeit, Kinderarbeitskonvention, Kermarbeitsrecbte, Weltgesellschaft, ILO

\section{Einführung}

Widerstand gegen Globalisierung beruht zu einem beachtlichen Teil auf der Annahme, diese würde zu einem sozialpolitischen ,race to the bottom" führen, so auch zu einem Abbau von Arbeitsrechten. Während alle Länder von der gemeinsamen Einhaltung von Kernarbeitsnormen profitieren würden, hat jedes einzelne Land einen Anreiz, diese Standards zu umgehen, um durch ,,social dumping“ kurzfristige Kostenvorteile zu erzielen. Diese Sorge hat Initiativen hervorgebracht, Formen von Global Governance zu etablieren. Einige der Umfassendsten waren die der International Labour Organization (Internationale Arbeitsorganisation, ILO). Die ILO ist als Sonderorganisation der Vereinten Nationen (seit 1946) für internationale Arbeitsrechtsfragen zuständig und damit ein zentraler Akteur der globalen Sozialpolitik. Einzigartig an der ILO ist, dass Regierungen, Arbeitgeberverbände und Gewerkschaften vertreten sind. Diese in den United Nations (Vereinten Nationen, UN) einmalige tripartistische Struktur stammt aus den Zeiten, als die ILO dem Völkerbund angehörte (Gründung der ILO 1919). 
Ende der 1990er erhob die ILO acht aus den fast zweihundert Arbeitsrechtskonventionen zu einer bindenden internationalen Arbeitsnorm. Diese acht grundlegenden Konventionen, auch fundamentale oder Kernarbeitskonventionen bzw. Kernarbeitrechte genannt (fundamental or core labour standards), wurden 1998 in der Declaration on Fundamental Principles and Rights at Work (fortan: Deklaration) festgeschrieben, mit dem Appell an die Mitgliedsländer, diese teilweise schon seit Jahrzehnten erlassenen Konventionen zu ratifizieren. Ziel war, den möglichen negativen sozialpolitischen Folgen der Globalisierung entgegenzuwirken. ${ }^{1}$ Mit dieser Konzentration auf Kernarbeitskonventionen kam die ILO wiederholten Aufforderungen nach, das Gewirr an Konventionen überblickbarer zu gestalten.

Dieser Beitrag stellt anhand einer Ereignisanalyse von 1973 bis 2005 dar, weshalb Länder eine der sozio-ökonomisch anspruchsvollsten Kernkonventionen ratifizieren: die Minimum Age Convention (Übereinkommen über das Mindestalter für die Zulassung zur Beschäftigung, ILO-Konvention 138, fortan: Kinderarbeitskonvention).

Die Ratifizierung internationaler Konventionen ist bedeutsam, da sie eine Anerkennung von weltgesellschaftlichen Normen darstellt. Die Konvention gegen Kinderarbeit ist ein besonders interessanter Fall, da sie im Gegensatz zu anderen, allgemein gehaltenen Maßgaben sehr konkrete Anforderungen an die Länder stellt, ihre Arbeitsmarktgesetze und -strukturen zu verändern. Die Ratifizierung der Kinderarbeitskonvention kann hohe ökonomische Kosten nach sich ziehen (Abu Sharkh 2000; Anker 1998). Würde Kinderarbeit, wie in der Konvention festgesetzt, abgeschafft werden, könnten arbeitsintensive Industrien einen erheblichen Wettbewerbsnachteil erfahren (Levison et al. 1996; Maskus 1997). In der internationalen Diskussion wird dies als Grund zitiert, weshalb diese ILO-Konvention zusammen mit der Konvention zur Vereinigungsfreiheit die am wenigsten ratifizierte Kernarbeitskonvention bleibt (diese Kinderarbeitskonvention weist 154 Ratifikationen auf, die Konvention zur Vereinigungs- und Verhandlungsfreiheit lediglich 150). ${ }^{2}$

Skeptiker verweisen darauf, dass viele internationale Abkommen nicht eingehalten werden und dass womöglich schon bei der Ratifizierung keine Absicht zur Vertragskonformität besteht. Jedoch ist die Nichteinhaltung von ILO-Konventionen mit höheren Kosten verbunden als die Umgehung anderer UN-Verträge. Die Einhaltung von ILO-Konventionen unterliegt einer besonders strengen Überwachung im UNSystem. Da die ILO-Struktur tripartistisch ist, schlägt sich dies auch im Aufsichtsapparat nieder, der aus drei Gruppen besteht: Regierungen, Arbeitgeberorganisationen und Gewerkschaften. Die tripartistische Struktur der ILO hat zur Folge, dass die verschiedenen Akteure die Vertragsbrüchigkeit der jeweils anderen reklamieren können. Die Länder unterliegen somit einem dreifachen Monitoring in Bezug darauf, ob sie mit den Konventionen konforme Gesetzgebungen etlassen.

Die Beobachtungen und Berichte aller drei Mitglieder werden von dem Committee of Experts on the Application of Conventions and Recommendations (Experten-

1 Die fünf Dimensionen dieser Kernarbeitsstandards sind: Verbot der Zwangsarbeit (Nr. 29 und 105); Vereinigungsfreiheit und Kollektivverhandlungen (Nr. 87 und 98); Beseitigung von Diskriminierung und Lohngleichstellung (Nr. 100 und 111); Abschaffung der Kinderarbeit (Nr. 138 und 182) (Greven/Scherrer (1998); ILO (1999); Kellerson (1998)).

2 Siehe http://www.ilo.org/ilolex/english/convdisp1.htm (Zugriff am 30.09.2009). 
komitee zur Anwendung von Konventionen und Empfehlungen, CEACR) angenommen. Dieser Sachverständigenausschuss für die Überprüfung der Befolgung von Konventionen veröffentlicht im Falle der Kinderarbeitskonvention alle zwei Jahre einen Annual Report, in dem Länder öffentlich gerügt werden, falls sie ihren vertraglichen Verpflichtungen nicht nachkommen (siehe Abu Sharkh 2007).

Zwar ist die ILO nicht imstande, bei Vertragsbrüchigkeit Sanktionen zu erlassen, jedoch bedeutet der Aufsichtsmechanismus, dass sich ein Land bei Zuwiderhandlung öffentlicher Kritik aussetzt. Neben Imageverlusten könnte das negative Folgen mit Blick auf die Verteilung von Entwicklungsgeldern und Handelssanktionen bedeuten. Ferner gelten in so genannten monistischen Rechtssystemen ratifizierte Konventionen als nationales Recht und sind somit von nationalen Akteuren einklagbar.

Im Zuge der Etablierung der World Trade Organization (Welthandelsorganisation, WTO) traten westliche Gewerkschaften und Non-Governmental Organizations (Nichtregierungsorganisationen, NGOs) für eine Sozialklausel ein (Abu Sharkh 2002; Charnovitz 1994; Frank 1999; Grote et al. 1998; Langhammer 1999; Liemt 1989; Malanowski 1997; OECD 1995; Sapir 1995; Wet 1998: 447; Windfuhr 1999; Zeeb 1994). Dies hätte Sanktionen bei Nichteinhaltung von Kernarbeitsrechten wie Kinderarbeit bedeutet, sofern ein Land die Kinderarbeitskonvention ratifiziert hatte. Laut internationalem Recht können Sanktionen gegen ein Land nur bei Bruch ratifizierter Konventionen erlassen werden, da nur die Ratifizierung völkerrechtliche Verbindlichkeiten schafft (Döhring 1999). Ein Land mit Kinderarbeit muss also mit Handelssanktionen nicht aufgrund der Kinderarbeit als solcher rechnen, sondern weil es die Kinderarbeitskonvention ratifiziert hat und nicht einhalten konnte.

Es kam zwar nach 1996 nicht einmal zur Bildung einer WTO-Arbeitsgruppe zum Thema Sozialstandards, jedoch konnten Entwicklungsländer aus zweierlei Gründen eine künftige Sozialklausel als Bedrohung werten. Zum einen hatten Entwicklungsländer bereits hinsichtlich der Intellectual Property Standards (TRIPS) die Erfahrung gemacht, dass entwickelte Länder ihre Agenda durchsetzen auf Kosten der Entwicklungsländer. Zum anderen wird Kinderarbeit von wichtigen internationalen Akteuren in diversen westlichen Handelsabkommen und Entwicklungshilfebedingungen thematisiert. Das europäische Schema allgemeiner Zollpräferenzen (APS) sieht besondere Vorteile für solche Länder vor, in denen bestimmte soziale und ökologische Mindestbedingungen, insbesondere die fundamentalen ILO-Konventionen, gesetzlich vorgeschrieben sind (Großmann/Koopmann 1994). Die USA hat eine Reihe von positiven Anreizen in verschiedenen Abkommen geschaffen, um Kernarbeitsnormen durchzusetzen. In der Caribbean Basin Initiative, dem Generalized System of Preferences (GSP) und der Overseas Private Investment Cooperation ist die Gewährung von Handelsvorteilen bzw. Entwicklungshilfe an das Bemühen der jeweiligen Länder gekoppelt, diese und andere Kernarbeitsnormen einzuhalten. Dies gilt für das ganze Land, also auch für die so genannten Export Processing Zones (Perez-Lopez 1987).

Die Gewährung von technischer Zusammenarbeit im Rahmen der USamerikanischen Agency for International Development (USAID) ist ebenfalls an die Einhaltung der Kernarbeitsnormen gekoppelt (Compa 1993). Die USA haben ihren Einfluss benutzt, um die Respektierung von Menschenrechten voranzutreiben, z.B. in Guatemala, indem wirtschaftliche Unterstützung zurückgehalten, Handelsbeschrän- 
kungen erlassen wurden oder sie die Vergabe internationaler Kredite verhinderten (Frey 1997).

Auch forderten wichtige internationale Akteure aktiv eine Sozialklausel. Das Europäische Parlament hatte bereits im Rahmen des General Agreement on Tariffs and Trade eine Sozialklausel avanciert (Wet 1998). Die permanente Delegation der EU forderte die WTO allerdings lediglich auf, stärker mit der ILO zusammenzuarbeiten und der ILO einen formellen Observer Status zu gewähren.

Ökonomisches sowie realpolitisches Kalkül würden es folglich nahelegen, dass Länder, die auch nur ein Minimum an Kinderarbeit aufweisen, diese Konvention nicht ratifizieren, da sie sich damit nach dem geltenden Völkerrecht potentiellen künftigen Handelssanktionen aussetzten. Je mehr Länder vom Handel abhängig sind, desto weniger wahrscheinlich sollte eine Ratifizierung sein. Anzumerken ist hier, dass jedoch bzgl. der Entwicklungshilfe, ceteris paribus, gelten sollte, dass Länder, die finanziell von der internationalen Gemeinschaft abhängig sind, die Kinderarbeitskonvention ratifizieren sollten, um zumindest ihre Bemühungen kundzutun, Kinderarbeit zu bekämpfen. Entwicklungshilfegelder werden im Rahmen der „technischen Zusammenarbeit“ z.T. auch vergeben, um Menschenrechtsmissstände zu verbessern, sofern sich ein Land glaubhaft darum bemüht.

Diese Annahmen lassen sich allerdings nicht bestätigen, da Länder trotz Kinderarbeit (selbst im formellen Sektor) und trotz Handelsabhängigkeit die ILOKinderarbeitskonvention ratifizieren. Weshalb Länder mit und ohne Kinderarbeit trotz solcher negativen Folgen internationale Konventionen mit humanitären Zielen ratifizieren, wurde in der Global-Governance-Forschung empirisch besonders im Rahmen der neoinstitutionalistischen Weltgesellschaftstheorie untersucht (s.u.). Seit über drei Jahrzehnten erforscht dieser Zweig der sonst theoretisch und empirisch oft wenig präzisen Globalisierungsforschung anhand quantitativer Analysen die Einflüsse globaler Normen und Akteure auf nationalstaatliches Verhalten. Gemäß dieser Theorie stellen internationale Konventionen einen wichtigen Bezugstahmen in dem Bestreben von Nationalstaaten dar, sich auf internationaler Ebene als legitime Akteure $z u$ positionieren. Internationale Regierungsorganisationen sowie NGOs transportieren Vorstellungen legitimer Formen von Nationalstaatlichkeit. Insbesondere Staaten, die erst vor kurzem ihre Unabhängigkeit erlangten, werden durch ein internationales Regelwerk sozialisiert. Die Logik der sozialen Legitimation („Angemessenheit“) und „Sozialisation" (Risse/Sikkink 1999) zur Erklärung nationalstaatlichen Verhaltens steht im Gegensatz zu rein funktionalistischen oder realpolitischen Erklärungsmodellen. Die Ergebnisse der hier vorgelegten Analyse bestätigen, dass ein Rekurs auf den Einfluss internationaler Organisationen und Deklarationen einen größeren Erklärungswert hinsichtlich der Ratifizierungswahrscheinlichkeit der Kinderarbeitskonvention beanspruchen kann als funktionalistische Erklärungen und ökonomische Abhängigkeitstheorien. Im Folgenden wird der Inhalt dieser Konvention vorgestellt und begründet. Der neoinstitutionalistische Strang der Weltgesellschaftsforschung wird als Bezugsrahmen für die Ratifizierungsanalyse gewählt. Im Folgenden werden internationale Definitionen und Initiativen zu Kinderarbeit ausgeführt (Teil 2). Teil 3 bettet die hier vorliegende Fragestellung in die Weltgesellschaftsforschung ein. Methodologische Erwägungen werden in Teil 4 und 5 behandelt. Die Diskussion der Ergebnisse bzw. das Fazit erfolgt in Teil 6 bzw. 7. 


\section{Kinder und Kinderarbeit in ILO-Konventionen}

Die hier betrachtete Kinderarbeitskonvention definiert „Kind“ als eine Person bis zum Alter von 14 Jahren. Die Altersgrenze darf zwar von jedem Land nach oben hin im Einklang mit den Schulgesetzen festgesetzt werden, muss jedoch mindestens 14 Jahre betragen. Die ILO-Konvention legt entsprechend fest, dass das Mindestarbeitsalter ,shall not be less than the age of completion of compulsory schooling and, in any case, shall not be less than 15 years” (Art. 2 (3)) ,and 13 years for light work“ (Art. 7 (2)). Der Begriff „Kinderarbeiter“ bezieht sich auf Kinder, die dem Arbeitsmarkt angehören und jederzeit für eine Erwerbsbeschäftigung zur Verfügung stehen. Im Gegensatz dazu werden als ,arbeitende Kinder" solche definiert, die lediglich Teilzeit arbeiten und bei denen ihre Arbeit in keinem Konflikt mit dem Schulbesuch steht. ${ }^{3}$

Obwohl Kinderarbeit bereits in früheren ILO-Diskussionen thematisiert wurde, stellen die beiden Konventionen zum Verbot von Kinderarbeit die jüngsten und am stärksten umstrittenen Kernarbeitskonventionen dar. ${ }^{4}$ Die sehr niedrige Ratifizierungsrate der ILO-Konvention von 1973 führte zur Verabschiedung einer weniger strikten Konvention im Jahre 1999. Die Worst Fiorms of Child Labour Convention (Übereinkommen über das Verbot und unverzügliche Maßnahmen zur Beseitigung der schlimmsten Formen der Kinderarbeit, ILO-Konvention Nr. 182) ist auf das Verbot besonders gefährlicher Arbeiten beschränkt. Diese Konvention war von dem Bemühen getragen, einen einfachen ersten Schritt zur Bekämpfung von Kinderarbeit zu ermöglichen. Das Bestreben der ILO bleibt es aber, Länder davon zu überzeugen, die umfassendere erste Konvention Nr. $138 \mathrm{zu}$ ratifizieren.

Neben den beiden ILO-Konventionen zum Verbot von Kinderarbeit existieren weitere Übereinkommen im Rahmen der UN. Die Geneva Declaration of the Rights of the Child beispielsweise wurde bereits 1924 erlassen. ${ }^{5}$ Dort findet sich jedoch kein Verbot von Kinderarbeit. Im Gegenteil, in Artikel 4 heißt es: „The child must be put in a position to earn a livelihood“". Das Recht auf Bildung ist erst in der Universal Declaration of Human Rights (Art. 26 (1)) zu finden. Im Jahr 1959 verabschiedete die Generalversammlung der Vereinten Nationen die Declaration of the Rights of a Child, die neben dem Recht auf Bildung (Prinzip 7) auch die Rechte des Kindes auf Schutz vor Ausbeutung, Vernachlässigung und Misshandlung beinhaltet (Prinzip 9). Im International Covenant on Economic, Social and Cultural Rights von 1966 wird zwar gefordert, dass "states should also set age limits below which the paid employment of child labour should be prohibited and punishable by law" (Art. 10 (3)), aber es wird kein Mindestalter festgelegt. 1989 schließlich wurde von der Generalversammlung die Convention on the Rights of the Child verabschiedet, die besagt, dass "states parties recognize the right of the child to education" (Art. 28). Dieses bindende Übereinkommen legt auch das Recht des Kindes auf Schutz vor ökonomischer Ausbeutung und schwerer oder

3 Siehe World Bank (2000: 49) für Details.

4 Dies ist erstaunlich, da die westliche sozialpolitische Gesetzgebung in vielerlei Hinsicht mit Kindern begann (Bequel/Myers 1995; Schäfer 1993), so in Preußen das Regulativ gegen Kinderarbeit von 1839. Dies ist international nicht der Fall.

5 Siehe http://www.un-documents.net/gdrc1924.htm (Zugriff am 04.04.2009). 
schädigender Arbeit fest (Art. 32). Jedoch bleibt auch diese Konvention vage in Bezug auf konkrete Alters- oder Arbeitsbeschränkungen.

Keine dieser Konventionen konkretisiert das Verbot von Kinderarbeit explizit. Vielmehr sind sie in Bezug auf Kinderarbeit so allgemein gehalten, dass alle Länder sie unterzeichnen können. Eine vorherige Ratifizierung anderer UN-Vereinbarungen ersetzt die Ratifizierung der Kinderarbeitskonvention somit weder de facto noch de jure. Die erste ILO-Kinderarbeitskonvention von 1973 explizierte schließlich das Verbot von Kinderarbeit bis zum 15ten Lebensjahr im internationalen Konsensus und ist für ratifizierende Staaten bindend (Abu Sharkh 2000, 2002).

\section{Weltgesellschaftsforschung}

In der Literatur über Kinderarbeit dominieren funktionalistische und ökonomische Erklärungsmodelle (Charnovitz 1994; Kulessa 1995; Leisink 1999; Scherrer 1998). Entwicklungshilfeliteratur, oft von technischen Experten aus internationalen Hilfsorganisationen geschrieben, hebt endogene ökonomische Faktoren hervor, etwa ein niedriges Bruttosozialprodukt für die Erklärung von Kinderarbeitstaten, teilweise in dem Verständnis, dass diese funktionalistischen Erklärungsmodelle auch für das Verhalten von Staaten vis á vis der Anerkennung von internationalen Kinderarbeitsnormen gelten (Anker 1998: 15; Archavanikul 1998; Bequel/Myers 1995; Canagarajah/Coulombe 1997; Compa 1993; ILO 1997; Fallon/Zafiris 1998; Grootaert 1998; Jennings 1999; Ravallion/Wodon 1999). Forschung im Bereich der Internationalen Bezichungen legt zudem nahe, dass Staaten Abkommen nur unterschreiben, wenn diese keinerlei Kosten verursachen, da die Vertragsverpflichtungen bereits erfüllt sind. Insofern würde eine Ratifizierung keine Richtungsänderung bedeuten, sondern lediglich ,reflections of underlying state preferences“ indizieren (Simmons/Hopkins 2005: 623; siehe auch Krasner 2000).

Im Gegensatz zu funktionalistischen oder realpolitischen Erklärungsmodellen hat die angelsächsische, neo-institutionalistische Weltgesellschaftsforschung in den letzten drei Dekaden gezeigt, dass globale Governance-Strukturen und deren in Konventionen kodifizierte Normen eine wichtige Rolle für die Erklärung von nationalstaatlichem Verhalten auf der internationalen Bühne spielen. Innerhalb der Forschungsdebatte zur nationalstaatlichen Regeleinhaltung ("Compliance") von international verankerten Normen kann dieser Forschungszweig in der Organisationsliteratur zum „Management-Ansatz“ bzw. in der Gobal-Governance-Terminologie zum „Befähigungsansatz" gezählt werden. ${ }^{6}$

Der Befähigungsansatz wird auch von der ILO selbst verfolgt (Liese 2005). Der Befähigungsansatz findet bei der ILO insbesondere in der Bekämpfung von Kinderarbeit durch die staatliche Implementierung relevanter Konventionen Anwendung. (Liese 2005). Senghaas-Knobloch (1999: 32, siehe auch 2005) argumentiert, dass „technische Zusammenarbeit als bewusst eingeschlagener Weg angesehen werden [...],

6 Verschiedene Konzepte der neo-institutionalistischen Weltgesellschaftsforschung sind der Organisationsforschung entlehnt. 
die ,Fähigkeit von Regierungen' (capacity of governments) zum Eingehen und Einhalten von verpflichtenden Übereinkommen zu Arbeits- und Sozialnormen zu erhöhen".

Das Management steht im Gegensatz zum Erzwingungsansatz, in dem etwa Sanktionen als ein geeignetes Mittel gesehen werden, Vertragskonformität bzw. die Einhaltung von „international regulatory agreements“ (Tallberg 2002: 609) zu erreichen. Der Befähigungsansatz hingegen setzt auf ,problem-solving strategy of capacity building, rule interpretation, and transparency, rather than coercive enforcement" (Tallberg 2002: 613). Anstelle von „Schiedssprüchen und formalen Regelbruchfeststellungen" wird auf der internationalen Ebene ein "Raum für die Diskussion von allgemeinen und fallspezifischen Problemen bei der Regeleinhaltung“ (Zürn 1997: 59) geschaffen.

Die vorliegende Untersuchung verortet sich deshalb theoretisch im Rahmen des von den UN-Organisationen selbst befolgten „Befähigungsansatzes“, um zu erforschen, inwieweit dieser in den letzten Dekaden erfolgreich war. Die neoinstitutionalistische Weltgesellschaftsforschung um John Meyer ist inhaltlich der geeignete Zweig des Befähigungsansatzes, da dieser theoretische Rahmen zudem den Vorteil hat, dass er sich anhand soziologischer Konzepte und Begrifflichkeiten umfassend auf Weltgesellschaftlichkeit bezieht (Wobbe 2000).

Unterschiedliche Konzeptualisierungen der Weltgesellschaft akzentuieren jeweils ökonomische, politische, kulturelle oder kommunikative Dimensionen (Bornschier 1996; Finnemore 1996; Forschungsgruppe Weltgesellschaft 1996; Robertson 1992). Der Ansatz von Meyer et al. (1997) greift über diese speziellen Dimensionen hinaus und versteht Weltgesellschaft als ein teils integriertes Arrangement von Organisationen und Prinzipien auf globaler Ebene, die korrektes nationalstaatliches Verhaiten spezifizieren. Das Konzept ist also nicht auf ökonomische, politische oder militärische Aspekte beschränkt. Methodologisch sieht Meyer Weltgesellschaft als eine soziale Konstruktion. Diese Konstruktion ist insofern eine Gesellschaft, als ein übergreifendes Wertesystem existiert. In diesem Wertesystem wird das Konzept des Individuums mit festgeschriebenen Menschenrechten verstanden (Finnemore 1996; Meyer et al. 1997; Wobbe 2000).

Einige weltgesellschaftliche Vorstellungen sind in diversen völkerrechtlichen Erklärungen wie der Allgemeinen Erklärung der Menscbenrecbte von 1948 sowie in völkerrechtlich bindenden internationalen Übereinkommen festgeschrieben. Verschiedene Mechanismen bewirken eine weltweite Verbreitung dieser kulturellen Modelle (Ramirez/Meyer 1998). Einen besonders wirksamen Mechanismus stellen die zahlreichen Regierungs- und Nichtregierungsorganisationen, vor allem die UN-Organisationen oder beispielsweise Greenpeace, deren Anzahl in den letzten 100 Jahren exponentiell zugenommen hat (Boli/Thomas 1999), dar. Diese internationalen Akteure transportieren Konzepte von globalen Arenen wie dem UN-System in die Länder (Meyer et al. 1997; Boli/Thomas 1999).

Sowohl inhaltlich als auch methodologisch stellt dieser Zweig des Befähigungsansatzes hier einen angebrachten Rahmen dar, da dieser Ansatz die in diesem Artikel verfolgte quantitative Herangehensweise favorisiert, um die Bedeutung internationaler Menschen- und Arbeitsrechtssysteme der UN nachzuweisen. Sich auf quantitative

7 Für einen frühen Ansatz siehe Burton (1972). 
Längsschnittsanalysen berufend, argumentieren Meyer et al. (1997), dass „many features of the contemporary nation-state derive from worldwide models constructed and propagated through global cultural and associational processes". Auf Länderebene stützen diese Modelle den Nationalstaat als einzig legitime Form der politischen Organisation (Meyer 1987; McNeely 1998). Innerhalb des Nationalstaats fördern sie die Emergenz von “Isomorphismen“: „surprisingly similar institutions of modernity (e.g., state forms, state services, educational systems) appear in all sorts of societies" (Meyer 1987: 42). Die formalen Organisationsstrukturen gleichen sich, obwohl sie nicht unbedingt die effizienteste Lösung für die Koordination von Aktivitäten darstellen (Meyer/Rowan 1977). Stattdessen fungieren sie als Mythen, die Legitimität in „Organisationsfeldern“ (DiMaggio/Powell 1991: 9) herstellen, welche den Referenzrahmen für Organisationen konstituieren.

Im Falle von Nationalstaaten werden diese Felder von anderen Staaten in der Weltgesellschaft konstituiert. Nationalstaaten werden durch internationale Organisationen in diese Felder eingebunden. Aufgrund dieser Prägung durch Organisationsfelder reflektieren nationale Gesellschaften exogene kulturelle Modelle in mehrfacher Weise (Ramirez/Meyer 1998: 64). Diese strukturelle Konformität zwischen Nationalstaaten geht über Gemeinsamkeiten hinaus, die durch ökonomische oder funktionale Rationalität, kulturelle und religiöse Traditionen oder Machtbeziehungen und internationale Abhängigkeiten erklärt werden könnten (Boli/Thomas 1999). Eine Ratifizierung der hier betrachteten Konvention wird in der Weltgesellschaftsliteratur als symbolische Anerkennung exogener, weltgesellschaftlicher Konzepte gedeutet.

Weltgesellschaftsforscher konnten den Einfluss exogener Faktoren etwa mit Blick auf den steigenden Anteil von Frauen an Universitäten (Bradley/Ramirez 1996), Schulsysteme und Einschulungsraten (Meyer et al. 1992), Antidiskriminierungsgesetzgebungen (Berkovitch 1999: 119; Ramirez/Meyer 1998: 70; Abu Sharkh 2009), das Frauenwahlrecht (Ramirez 2000; Ramirez et al. 1997), Wohlfahrtsstaats- und Landreformen (Thomas/Lauderdale 1988) sowie Verfassungen (Boli 1987) nachweisen. Im Falle der Gesetzgebung für Kinder haben Boli und Meyer gezeigt, wie die Ideologie einer besonderen Lebensphase der "Kindheit“ in Verfassungen und die daraus folgenden gesetzlichen Beschränkungen der Erwerbsbeteiligung von Kindern "legitimating ideas dominant in the world system at the time of their creation" (1987: 220) reflektieren. Jedoch haben Neo-Institutionalisten weder systematisch die Ratifizierung von Kernarbeitsnormen im Allgemeinen noch Kinderarbeitskonventionen im Besonderen analysiert. ${ }^{8}$

Die Ratifizierung stellt gemäß des Befähigungsansatzes den entscheidenden ersten Schritt dar, in dem sich souveräne Staaten verpflichten, den Weg internationaler Normumsetzung zu beschreiten. Die Analyse des Ratifizierungsverhaltens der Kinderarbeitskonvention zeigt, welche Faktoren einen solchen ersten Schritt begünstigen, und erweitert somit die Weltgesellschafts- und Global Governance-Forschung.

8 Es besteht jedoch Literatur zu Aspekten von Arbeitsrechten: In einem Versuch, GenderRegimes zu klassifizieren, beleuchtet Orloff (1998) die Auswirkung von wohlfahrtsstaatlichen Bestimmungen auf Geschlechterbeziehungen. Die Institutionalisierung der Arbeitsunfallversicherung für Unfälle im verarbeitenden Gewerbe wurde von Grattet (1998) dargelegt (s. dazu den Beitrag von Leisering/Liu 2010 in diesem Heft). 


\section{Variablen, Hypothesen und Daten}

Aus der Perspektive der neoinstitutionalistischen Weltgesellschaftstheorie verpflichten sich Nationen aus Gründen der Selbstlegitimation in der internationalen Gemeinschaft auf bestimmte Normen. Je stärker Staaten in die Netzwerke der internationalen Gemeinschaft eingebunden sind, umso mehr Druck, so wird angenommen, sind sie ausgesetzt, internationale Normen zumindest formal anzuerkennen, unabhängig von den tatsächlichen Gegebenheiten im Land (Meyer et al. 1997: 154ff.). HafnerBurton et al. (2008) konnten beispielsweise eindrücklich zeigen, dass repressive Staaten mit genauso hoher Wahrscheinlichkeit Menschenrechtskonventionen unterzeichnen wie nicht-repressive Staaten.

Die neoinstitutionalistische Weltgesellschaftsliteratur sieht internationale Organisationen als Träger weltgesellschaftlicher Normen. Global verlinkte Nationen, sowohl durch internationale NGOs (Wotipka/Ramirez 2008) sowie durch internationale Regierungsorganisationen (Wotipka/Tsutsui 2008), haben eine signifikant höhere Wahrscheinlichkeit, Menschenrechtsabkommen zu ratifizieren. Übertragen auf die zu den Menschenrechtskonventionen gezählten Kernarbeitskonventionen impliziert dies folgende Hypothese:

Hypothese 1: Mitgliedschaft in internationalen Organisationen erhöh wesentlich die Wahrscheinlichkeit, die Kinderarbeitskonvention zu ratifizieren.

Doch welche Organisationen sind am effektivsten? Gamson (1997: 95) sprach als erster von dem "success of the unruly“. Damit meinte er, entgegen verbreiteter Ansichten, dass ,unruly groups, those that use violence, strikes, and other constraints, have better than average success" (Gamson 1997a: 95). Auch Tronto (1991: 1990) argumentiert, dass ,a radical fringe is essential for more mainstream accomplishments, rather than being a nuisance or embarrassment." McAdam et al. (1996) nennen diese den "radical flank effect“. Da NGOs nicht in einem diplomatischen Korsett gefesselt sind, steht ihnen ein größeres Repertoire an Aufsehen erregenden Möglichkeiten zur Verfügung. Man denke beispielsweise an die unterschiedlichen Möglichkeiten, zwischen der NGO, Greenpeace und dem United Nations Environment Programme öffentliche Aufmerksamkeit zu forcieren. Dies legt eine weitere Hypothese nahe:

Hypothese 2: NGOs üben einen stärkeren Druck auf die Ratifizierungswahrscheinlichkeit aus als internationale Regierungsorganisationen.

Ereignisse auf der globalen Ebene können ebenfalls das Ratifizierungsverhalten einzelner Länder beeinflussen. Wotipka und Ramirez (2008) zeigen beispielsweise, dass Frauenkonferenzen, wie in Beijing, einen starken Effekt auf die Ratifizierung der Convention on the Elimination of All Forms of Discrimination against Women (CEDAW) ausüben. Frauenkonferenzen werden als externe, weltgesellschaftliche Einflussgrößen konzeptualisiert, als externer, internationaler „Schockeffekt“. Da Frauenkonferenzen die Ratifizierung von CEDAW vorantreiben, wird hier vermutet, dass die IL.ODeklaration im Rahmen der Weltkonferenz zu fundamentalen Arbeitskonventionen solch einen „Schockeffekt" darstellt und neue Ratifizierungen veranlasst. 
Hypothese 3: Im Jahr der ILO-Deklaration 1998 und im darauf folgenden Jahr stieg die Ratifizierungswahrscheinlichkeit stark an.

Ein weiterer Indikator für eine Einbindung in die moralische Ordnung der Weltgesellschaft ist die Anzahl der sonstigen Kernarbeitskonventionen, die ein Land ratifiziert hat. Diese Konventionen sind nicht alle miteinander thematisch verknüpft. Die Ratifizierung einer der acht Konventionen ist deshalb, bis auf eine Ausnahme, inhaltlich unabhängig von der Ratifizierung der anderen Konventionen. Jedoch könnte die Ratifizierung einzelner Konventionen des Konventionenpakets als ein starkes Signal gedeutet werden, die Weltnorm der Kernarbeitsstandards insgesamt aufrecht zu halten, einschließlich des Verbotes von Kinderarbeit. Wotipka und Ramirez (2008) konnten beispielsweise nachweisen, dass Staaten, die andere Menschenrechtskonventionen ratifiziert haben, auch wenn diese nichts mit Frauenrechten zu tun haben, eher dazu geneigt sind, auch CED $\Lambda \mathrm{W}$ zu ratifizieren.

Hypothese 4: Die Ratifizierung der anderen sieben Kernarbeitskonventionen erhöht die Ratifizierung der Kinderarbeitskonvention signifikant.

Die in den Hypothesen vorkommenden Standardfaktoren der Weltgesellschaftsforschung messen nationalstaatliches Legitimationsbestreben. Im Fall von $\Lambda$ rbeitsrechtkonventionen ist das Legitimationsbedürfnis jedoch vermutlich nicht allein von der Zugehörigkeit $\mathrm{zu}$ internationalen Organisationen geprägt. Eine wichtige Kontrollvariable ist deshalb die politische Ausrichtung des Systems. Die Ost-West-Rivalität spornte sozialistische Länder an, ihre moralische und ideologische Überlegenheit durch die formale Anerkennung von Arbeitsrechtkonventionen zu demonstrieren, sowohl um ihr System gegenüber ihrer eigenen Bevölkerung zu rechtfertigen als auch um die Anerkennung von Staaten im globalen Süden zu gewinnen. Auch führte das Blockverhalten vermutlich dazu, dass südliche Satellitenstaaten das Ratifizierungsverhalten der Sowjetunion nachahmten, die die Kinderarbeitskonvention bereits 1979 ratifiziert hatte. Sozialistische Länder werden somit wahrscheinlich ihr Ratifizierungsverhalten aufeinander abstimmen.

Das westliche Pendant zur ideologiebezogenen Ratifizierungshypothese in Bezug auf die sozialistischen Länder ist ebenfalls als eine Kontrollvariable in das Modell eingebaut. Vermutet wird, dass demokratische Länder aufgrund ihrer ideologischen Nähe zur Norm der Selbstverwirklichung durch Bildung Kinderarbeit symbolisch durch Ratifizierung delegitimieren.

Allein ökonomisch-rationalistisch argumentierend legen manche Beobachter nahe, dass Länder, in denen viel Kinderarbeit herrscht, keinen Anreiz haben, die Kinderarbeitskonvention zu unterschreiben. Deshalb ist eine wichtige Kontrollvariable die Kinderarbeitsrate eines Landes. Kinderarbeit wird im Modell als Prozentsatz der arbeitenden Kinder der Alterskohorte der 10- bis 14-Jährigen gemessen.

Ökonomisch-rationalistisch gesehen dürfte Kinderarbeit insbesondere bei Handelsabhängigkeit die Ratifikationswahrscheinlichkeit schmälern. Wie oben ausgeführt, können laut internationalem Recht nur Handelssanktionen gegen ein Land erlassen werden, wenn es gegen internationale Konventionen verstößt, die es selbst ratifiziert hat. Nicht Kinderarbeit per se, sondern Kinderarbeit trotz Ratifizierung der Kinderarbeitskonvention öffnen ein Land für potentielle Handelssanktionen. Handelsabhängigkeit sollte laut dieser Logik deshalb einer Ratifikation entgegenwirken. Handelsab- 
hängigkeit wird gemessen als Prozentsatz von Import und Export am Bruttoinlandsprodukt (BIP). Sehr arme Länder haben oft einen hohen Prozentsatz an Kinderarbeit und wenig institutionelle Kapazitäten, dies zu beheben. Vermutet wird deshalb, dass wenig entwickelte Länder zögern, qua Ratifikation eine Verpflichtung einzugehen, die sie nicht halten können. Der Reichtum eines Landes wird operationalisiert als Einkommen gemessen in Bruttoinlandsprodukt pro Kopf. Alle diese Variablen entstammen den World Development Indicators der World Bank.

Die Daten zur Ratifizierung der Kinderarbeitskonvention sowie der anderen Kernarbeitskonventionen stammen aus der Online-ILO-Datenbank ILOLEX. Die unabhängigen Variablen werden wie in der Weltgesellschaftsforschung üblich operationalisiert. So wurde Mitgliedschaft in internationalen Organisationen anhand der Veröffentlichungen der Union of International Associations kodiert. Der externe Schockeffekt der ILO-Deklaration 1998 wird durch eine dichotome Variable für das Jahr der Deklaration sowie das darauf folgende Jahr markiert. Die Werte der sozioökonomischen Ländervariablen sind den World Development Indicators entnommen.

\section{Methoden und Analyseeinheit}

Für eine angemessene Methode zur Untersuchung der Ratifizierung von Kinderarbeitskonventionen wurde eine Ereignisanalyse für den Zeitraum von 1973 bis 2005 durchgeführt. Dieses Vorgehen lehnt sich an verwandten Arbeiten von Weltgesellschaftsforschern zur Untersuchung ähnlicher Fragen an (Tsutsui 1998; Hironaka 1997; Wotipka/Ramirez 2008; Wotipka/Tsutsui 2008; Hafner-Burton et al. 2008).

Die Ratifizierungsmuster der Kinderarbeitskonvention lassen sich am besten in einer zeitsensitiven Analyse einfangen. Zum einen ist es notwendig, dass die Einheiten über den Zeitraum hinweg beobachtbar sind, seitdem die Konvention verabschiedet wurde und zur Ratifizierung offen steht. Zum anderen sprechen mehrere methodische Erwägungen für die Durchführung einer solchen Analyse. Der Hauptgrund besteht in der Vermeidung eines Spezifizierungsfehlers. Längsschnittanalysen beheben zu einem beachtlichen Teil die konfundierenden Effekte, wenn zeitinvariante Variablen im Modell ausgelassen werden (Hannan/Tuma 1979: 1-8). Ähnlich wie bei anderen Typen von Regressionsanalysen erlaubt die Analyse von Standardabweichungen die Unterscheidung zwischen zufälligen und signifikanten Variationen in den Daten (Hannan/Tuma 1979).

Von den verschiedenen Möglichkeiten einer Längsschnittanalyse ist die Ereignisanalyse hier besonders angemessen, da untersucht werden soll, welche Faktoren ein Ereignis beschleunigen. Das Ereignis ist in diesem Fall die Ratifizierung. Die abhängige Variable ist somit die Zeitspanne, bis dieses Ereignis stattfindet.

Ein Problem von Ereignisanalysen besteht in der Zensur des betrachteten Zeitraums. Daten sind links zensiert, wenn das Ereignis vor dem Beobachtungszeitraum hätte stattfinden können. Eine solche Zensur würde beachtliche methodische Probleme aufwerfen. Die Ratifizierungsdaten sind jedoch nicht links zensiert, da Länder die Konvention erst nach deren Erklärung im Jahr 1973, dem Beginn des Beobachtungszeitraums, ratifizieren konnten. Da die Kinderarbeitskonvention 1973 verabschiedet wurde, wird dieses Jahr als Beginn des Analysezeitraums gesetzt. Für Natio- 
nen, die erst nach 1973 ihre Unabhängigkeit erlangt haben, beginnt die Ereignisanalyse erst mit ihrer nationalstaatlichen Souveränität. Die Rechtszensur der Daten, also der Sachverhalt, dass nicht alle Länder innerhalb des Beobachtungszeitraums die Konvention ratifiziert haben, birgt kein methodisches Problem. Dies trifft ohnehin nur für 20\% der Länder zu. Aufgrund der Verfügbarkeit von Daten endet der Analysezeitraum 2005.

Verschiedene Modelle stehen bei der ,limited dependent variable technique“ der Ereignisanalyse zur Verfügung. Semi-parametrische Modelle wie das Cox-Modell haben den Vorteil, dass für die Zeitabhängigkeit von Ratifikation keine bestimmte funktionale Form angenommen wird und somit die Verteilung nicht artifiziell in eine bestimmte Form gepresst wird. Semi-parametrische Modelle sind jedoch wesentlich weniger effizient. Dies hat zur Folge, dass es schwerer ist, für die unabhängigen Variablen die statistische Signifikanz-Hürde zu überwinden. Trotz dieser höheren Hürde bei der Verwendung des Cox-Modells behielten alle Variablen, die weltgesellschaftlichen Einfluss messen, ihre Signifikanz mit einer Ausnahme: der Deklaration.

Dies liegt vermutlich daran, dass Cox-Modelle multiple ties, also viele Ereignisse im selben Jahr, nicht handhaben können (Cleves et al. 2008). Wie in Hypothese 3 dargelegt und von den Ergebnissen jedoch bestätigt wird, ist eine markante Häufung der Ratifizierungen im Jahr der Verabschiedung der Deklaration sowie im darauf folgenden zu verzeichnen.

Deshalb wird hier ein parametrisches Modell verwendet. Da keine theoretische Begründung für die Annahme einer besonderen funktionalen Form vorliegt, wird ein parametrisches Modell verwendet, das keine besonderen Ausschläge über die Zeit annimmt: das exponentielle Modell. Dieses Modell wird auch von anderen weltgesellschaftlichen Untersuchungen nahegelegt (siehe beispielsweise Wotipka/Ramirez 2008 im Text). Diese Entscheidung wurde auch durch zusätzliche Tests, wie den AIC-Test (siehe Cleves et al. 2008), validiert.

Die Analyseeinheit ist die Nation. Ereignisanalysen auf der nationalstaatlichen Ebene wurden bereits etwa von Tsutsui (1998) hinsichtlich internationaler Verträge oder von Hironaka (1997) hinsichtlich internationaler Auseinandersetzungen durchgeführt. Nur Nationalstaaten, die der ILO angehören, werden einbezogen, da nur diese die ILO-Konventionen ratifizieren können. De facto macht dies jedoch keinen Unterschied, da alle Länder mit Ausnahme einiger Kleinststaaten wie Tonga oder umstrittener Gebiete wie Taiwan ILO-Mitglieder sind.

Die Analyse beruht daher nicht auf einer Stichptobe im strikten Sinne, da so gut wie alle Nationalstaaten eingeschlossen sind, vorausgesetzt, die Daten sind verfügbar. Die verwendeten Daten der World Bank werden meistens von den entsprechenden spezialisierten UN-Organisationen zusammen mit den nationalen, statistischen Ämtern in den Ländern erhoben bzw. in die UN-Datenbanken eingespeist. Der Einbezug von Ländern in die Analyse ist damit an die Frage gebunden, welche Länder aus welchen Gründen keine Daten liefern.

Die problematischste Variable stellt in dieser Hinsicht das Einkommen pro Kopf dar. Zum einen können diese Werte zu niedrig angesetzt sein, da sich nur Länder mit einem relativ niedrigen Pro-Kopf-Einkommen für die begehrten günstigen Kredite im Rahmen von Official Development Assistance qualifizieren (Nuschler 1996). Entwicklungsländer haben somit einen Anreiz, das Bruttoinlandsprodukt pro Kopf 
systematisch zu untertreiben. Zweitens ist dies die Variable mit den meisten fehlenden Werten. Dies ist vor allem für folgende Ländertypen in der World-DevelopmentIndicator-Datenbank der World Bank ausgeprägt: (1) kleine Inselstaaten, da sie vermutlich über keine ausreichende staatliche Infrastruktur für die Erhebung von Daten verfügen, (2) Bürgerkriegsländer mit einer ungenügenden staatlichen Infrastruktur und (3) sehr reiche Ölländer wie Qatar oder Kuwait, die wenig Interesse an Hilfeleistungen der internationalen Gemeinschaft haben.

\section{Analyse und Diskussion}

Bis zur ersten Ratifizierung der Kinderarbeitskonvention verstrichen zwei Jahre. Dies ist ungewöhnlich, da die feierliche Verabschiedung einer Konvention oft eine anfängliche Ratifizierungseuphorie nach sich zieht. Kuba (Ratifizierung: 07.03.1975) war das erste Land, das diese Konvention ratifizierte. Die offizielle Kinderarbeitsrate in Kuba war zu diesem Zeitpunkt 1.5\%. Doch da diese Zahl nur arbeitende Kinder im formellen Sektor beinhaltet, könnte die tatsächliche Kinderarbeitsrate um einiges höher gelegen haben. Unabhängig von der genauen Kinderarbeitsrate sollte jegliche Kinderarbeit eine Ratifizierung bremsen, da jedes einzelne arbeitende Kind einen Bruch der unterzeichneten Konvention darstellt. Die Konvention spezifiziert nicht, dass ein geringes Ausmaß an Kinderarbeit zulässig ist, sondern verbietet Kinderarbeit prinzipiell. Somit war die Ratifizierung nicht Anzeichen einer Arbeitsmarktstruktur, die keine Ratifizierungskosten verursachen würde, wie realpolitische Argumente suggerieren würden. Gerade die letzen Prozente an Kinderarbeit können besonders schwer auszumerzen sein, da sie in für die Regierung nur schwer zugänglichen Industriezweigen stattfindet. Das nächste ratifizierende Land war Libyen (Ratifizierung: 16.06.1975). Auch Libyen kann als sozialistisch gelten, obwohl Gaddafi mit der "Third Universal Theory" eine eigene Variante verfolgte, in der Kommunismus mit Islam vereint werden sollte. Auch das dritte ratifizierende Land, Rumänien (Ratifizierung: 19.11.1975), war sozialistisch und zudem der osteuropäische Staat, der zu diesem Zeitpunk die höchste Kinderarbeitsrate Osteuropas aufwies.

Die frühen Ratifizierungen scheinen somit in zweifacher Hinsicht von ideologischen Gründen getragen zu sein. Zum einen spiegeln sie die staatliche Lenkung von Arbeitskraft in die dafür vorgesehenen, nämlich erwachsenen, Lebensphasen im sozialistischen System wider und das damit einhergehende Verbot von Kinderarbeit. Interviews mit ILO-Mitarbeitern legen zudem nahe, dass gerade diese sozialistischen Außenseiter sich auf internationaler Ebene legitimieren wollten. Das vierte "Land“ waren die Bundesrepublik Deutschland (Ratifizierung: 07.03.1975) und die Deutsche Demokratische Republik. Vermutlich spielte die Ost-West-Rivalität hier ebenfalls eine Rolle.

Abbildung 1 zeigt die Kaplan-Meier-Funktionen von Ländern, die der Organisation for Economic Co-operation and Development (Organisation für wirtschaftliche Zusammenarbeit und Entwicklung, OECD) angehören, versus Staaten, die der OECD nicht angehören. Der Kaplan-Meier-Schätzer dient zum Schätzen der Wahrscheinlichkeit, dass bei Ländern das Ereignis „Ratifizierung“ innerhalb eines Zeitintervalls nicbt eintritt. Die Y-Achse stellt die „Survival“-Wahrscheinlichkeit dar. Die Kurven zeigen 
die hohe „Überlebenswahrscheinlichkeit", in ereignisanalytischer Terminologie, der nicht ratifizierenden Staaten. Bis in die 1990er Jahre ratifizierten nur wenige Länder, auch unter den entwickelten Industriestaaten, diese Konvention in den ihrer Erklärung 1973 folgenden Dekaden. Dies stellt einen beachtlichen Unterschied zu dem Ratifizierungsverhalten bzgl. anderer Kernkonventionen, z.B. der zu Diskriminierung, dar. Keine Nation zog die beteits vollzogene Ratifizierung jedoch zurück.

Die als Unterscheidungsmerkmal gewählte OECD-Zugehörigkeit ist, wie jede Klassifizierung, nicht in allen Fällen triftig; so gehören auch Mexiko und Südkorea zur OECD. Jedoch entspricht die Klassifizierung „OECD-Zugehörigkeit" in etwa dem allgemeinen Verständnis von ,westlichen“ Ländern. OECD-Länder setzen sich in ihrer ökonomischen Struktur von Entwicklungsländern ab. Wäre beispielsweise nur Einkommen der Unterscheidungsfaktor, würde dies die wichtigen Unterschiede zwischen reichen westlichen und ebenfalls einkommensstarken Ölstaaten verschleiern (siehe unten).

In der Gruppe der OECD-Länder finden sich deutlich weniger Ratifizierungsverweigerer als in der Gruppe der Nicht-OECD-Staaten. Eine Erklärung wäre, dass spätestens seit den 1980ern ohnehin kaum noch Kinderarbeit in OECD-Staaten existiert, außer bei einigen Immigrantengruppen. In den OECD-Staaten lag die durchschnittliche Kinderarbeitsrate unter 1\%. Dies scheint die realpolitischen Argumente der internationalen Beziehungen zu bestätigen, dass Länder nur bereit sind, solche internationalen Abkommen zu unterzeichen, die keine Kosten nach sich ziehen, da sie deren vertragliche Vereinbarungen bereits erfüllt haben. Auch scheint dies Argumente aus der Entwicklungsliteratur zu bestätigen, dass die Einstellung zu Kinderarbeit eine Funktion des Bruttosozialprodukts pro Kopf ist. Zur Klärung dieser Vermutungen ist es jedoch hilfreich, zwischen reichen OECD- und Nicht-OECDLändern, etwa den Ländern der Organization of the Petroleum Exporting Countries (OPEC), zu unterscheiden. Weiterführende Analysen zeigen, dass OPEC-Staaten, obwohl sie ebenfalls Kinderarbeitsraten von unter 1\% haben, eine signifikant geringere Wahrscheinlichkeit als ihre westlichen Einkommensgenossen aufweisen, die Kinderarbeitskonvention zu ratifizieren. Dies deutet darauf hin, dass Wertmuster und andere nicht-ökonomische Faktoren ebenfalls eine Rolle spielen bei der symbolischen Anerkennung der Rechte von Kindern.

Eine weitere Auffälligkeit ist, dass OECD-Länder die Ratifizierung in Schüben zu vollziehen scheinen, was in Abbildung 1 dazu führt, dass sie merklich von der prognostizierten Funktion divergieren. In manchen Jahren ratifiziert eine Reihe von OECD-Staaten die Konvention, in anderen Jahren allerdings kein einziger, da Kinderarbeit während dieser Jahre vermutlich nicht auf der politischen Agenda stand. Ein merklicher Ratifizierungsschub ist Anfang der 1990er Jahre zu beobachten. Etliche Staaten und Territorien erlangten ihre Unabhängigkeit und waren bestrebt, sich schnell auf der internationalen Bühne zu legitimieren. Eine Reihe von ehemaligen sowjetischen Republiken wie Tadschikistan ratifizierten bei Erlangung der Unabhängigkeit fast alle Kernkonventionen, inklusive der Kinderarbeitskonvention. Im Falle Tadschikistans wurde lediglich die weit verbreitete Konvention zur Gefangenenarbeit ausgelassen. 


\section{Abbildung 1: Kaplan-Meier-Schätzungen der Survivor-Funktion der Nicht- Ratifizierung der ILO-Kinderarbeitskonvention 1973 nach OECD- Mitgliedschaft}

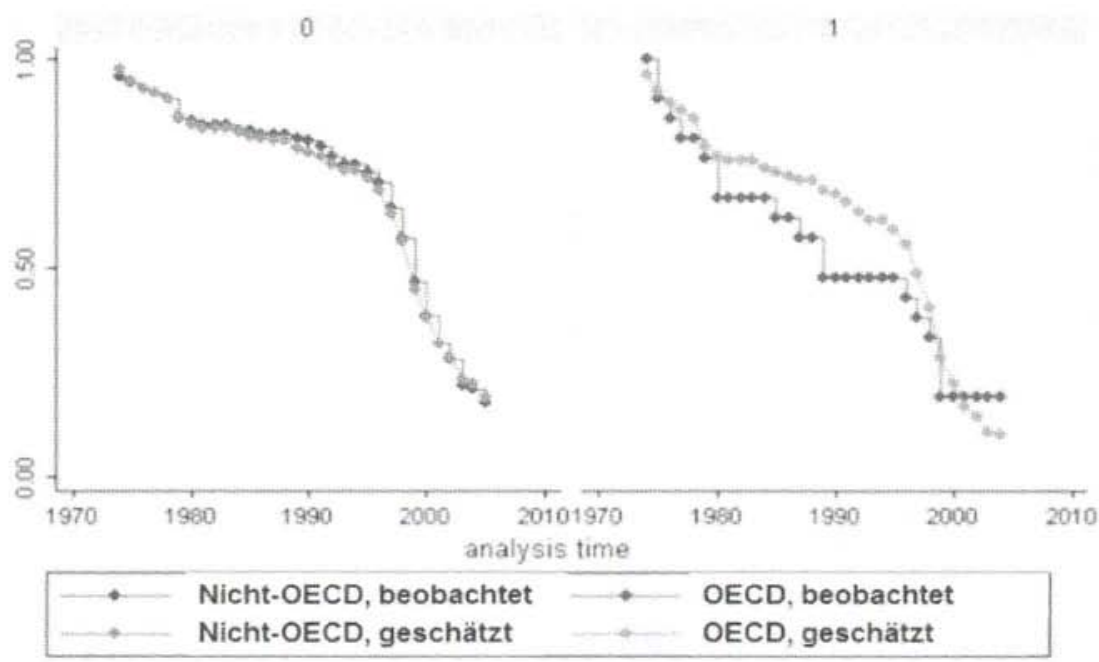

Quelle: ILOLEX, eigene Analysen

Der zweite Schub fand kurz vor der Jahrtausendwende statt. Der in Abbildung $1 \mathrm{zu}$ beobachtende dramatische Abfall der Nicht-Ratifizierer genau um den Zeitpunkt der Deklaration der Kernarbeitsnormen (1998) herum ist vermutlich auf den Druck der ILO zurückzuführen, die ihre Mitgliedsstaaten dazu anhielt, die acht Kernarbeitskonventionen zu ratifizieren. Innerhalb von fünf Jahren verdoppelten sich die Ratifizierungen. Die ILO-Deklaration von 1998 war insofern eine beachtliche Erfolgsgeschichte. Wie Abbildung 1 nahelegt und ein Log-Rank-Test bestätigt, bestehen jedoch signifikante Unterschiede zwischen OECD- und Nicht-OECD-Ländern bezüglich der Wirksamkeit dieses weltgesellschaftlichen, externen „Schocks“. Für beide Gruppen scheint die Deklaration einen entscheidenden Schub nach vorangegangener Stagnation darzustellen. Für Nicht-OECD-Staaten ist die 1998er-Deklaration jedoch einflussreicher, vermutlich weil diese Staaten in ein weniger dichtes Netzwerk von wertetransportierenden Organisationen eingebunden sind.

Die Hazardraten sind in Tabelle 1 dargestellt. Die Hazardfunktion $\mathrm{h}(\mathrm{t})$ ist definiert als die Wahrscheinlichkeit, mit der die Ratifizierung zum Zeitpunkt $\mathrm{T}$ eintritt unter der Voraussetzung, dass sie bis zum Zeitpunkt t noch nicht eingetreten ist. Die Gleichung in diesem Fall lautet:

$$
h(t) d t=P(t<T<t+d t \mid T>t)=\frac{f(t) d t}{S(t)}=-\frac{S^{\prime}(t) d t}{S(t)}
$$


Die Hazardraten im Modell sind so zu lesen, dass Werte über 1 sich positiv auf die Ratifizierungswahrscheinlichkeit auswirken und Werte unter 1 negativ. Die Modelle sind nach der Logik zunehmender Berücksichtigung von nationalen ideologischen und weltgesellschaftlichen Faktoren aufgebaut. Modell 1 berücksichtigt funktionalistisch-ökonomische, realpolitische und weltgesellschaftliche Erklärungen. Wie in der Entwicklungsliteratur nahegelegt wird, haben einkommensarme Länder eine signifikant geringere Wahrscheinlichkeit, die Konvention zu ratifizieren. Jedoch scheint dies nicht allein durch die Kinderarbeitsrate im Land bedingt zu sein. Arme Länder mit einer hohen Kinderarbeitsrate, wie viele afrikanische Staaten, ratifizieren die Konvention nicht mit geringerer Wahrscheinlichkeit als einkommensschwache Nationen mit niedrigen Kinderarbeitsraten in Lateinamerika.

Auch scheinen Abhängigkeitsverhältnisse, wie realpolitische Erwägungen sie suggerieren würden, eine untergeordnete Rolle zu spielen. Weder eine Abhängigkeit von Entwicklungshilfe noch eine von Handel stellen signifikante Faktoren in der Bestimmung der Ratifizierungswahrscheinlichkeit dar.

Ideologische Faktoren spielen dagegen eine bedeutende Rolle. Länder mit (ehemals) sozialistischen Ideologien haben eine signifikant größere Wahrscheinlichkeit ( $90 \%$ größer!), diese Konvention zu ratifizieren, was schon die bisherigen Ausführungen nahelegten. Interessanterweise ratifizierte die Sowjetunion die Konvention erst im Jahr 1979. Sozialistische Paria-Staaten wie Kuba, Libyen und Rumänien hatten somit vor dem vermeintlichen ideologisch tonangebenden großen Bruder die Konvention ratifiziert. Das Ratifizierungsverhalten des Ostblocks im weitesten Sinne war somit nicht streng von der Sowjetunion geleitet. Demokratien haben überraschenderweise keine sich durch alle Modelle durchziehende signifikant größere Wahrscheinlichkeit, diese politisch korrekte Konvention zu ratifizieren. Außer in Modell zwei verfehlt die Demokratievariable die .05-Signifikanzhürde.

Das staatliche Organisationsprinzip ist in allen Modellen signifikant: Föderale Staaten ratifizieren diese, und vermutlich andere arbeitsrechtliche Konventionen, mit etwa halb so großer Wahrscheinlichkeit, da arbeitsrechtliche Fragen teilweise in den Kompetenzbereich der Bundesstaaten fallen.

Die ehemaligen Ostblockstaaten stellen jedoch in zweifacher Hinsicht eine Anomalie dar. Erstens haben die Staaten, die zur Union der sozialistischen Sowjetrepubliken gehörten, die Ratifizierungen, die von Moskau getätigt wurden, unterschiedlich gehandhabt. Die baltischen Staaten beispielsweise haben die Kinderarbeitskonvention nach ihrer Unabhängigkeit erneut ratifiziert, während Weißrussland das Datum der Ratifizierung durch die Sowjetunion beibehalten hat. Die neuen mittel- und osteuropäischen Staaten wie Serbien haben die Konvention erst nach der Deklaration von 1998 ratifiziert. Da die territoriale Desintegration von Osteuropa einen historischen Sonderfall darstellt, wurden alle in der Tabelle aufgefühtten Regressionen auch ohne ehemalige kommunistische Staaten durchgeführt, um die Robustheit der Ergebnisse zu prüfen. Diese Regressionen erzielten trotz einer kleineren Stichprobe sehr ähnliche Resultate, insbesondere was den Einfluss von weltgesellschaftlichen Faktoren betraf. 
Tabelle 1: Hazardraten der Ratifizierung der ILO-Kinderarbeitskonvention (1973-2005)

\begin{tabular}{|c|c|c|c|c|c|}
\hline Variable & Modell 1 & Modell 2 & Modell 3 & Modell 4 & Modell 5 \\
\hline $\begin{array}{l}\text { Weltgesellschaftliche Faktoren } \\
\text { Internationale Regierungsorga- } \\
\text { nisationen (log) }\end{array}$ & & $\begin{array}{l}3.15 * * * \\
(1.19)\end{array}$ & $\begin{array}{l}1.23 \\
(.58)\end{array}$ & & \\
\hline $\begin{array}{l}\text { Internationale NGOs (log) } \\
\text { Kernarbeitsdeklaration von } \\
1998 \\
\text { Ratifizierung der Kernarbeits- } \\
\text { konventionen }\end{array}$ & $\begin{array}{l}2.84^{* * *} \\
(.67)\end{array}$ & & $\begin{array}{l}2.65^{* * *} \\
(.75)\end{array}$ & $\begin{array}{l}2.59^{* * *} \\
(.61) \\
4.93^{* * *} \\
(1.45)\end{array}$ & $\begin{array}{l}1.67^{* *} \\
(.41) \\
4.63^{* * *} \\
(1.36) \\
1.67^{* * *} \\
(.17)\end{array}$ \\
\hline $\begin{array}{l}\text { Internationale ökonomische } \\
\text { Abhängigkeit } \\
\text { Handel (\% des Bruttosozial- } \\
\text { produkts) } \\
\text { Entwicklungshilfe (\% des } \\
\text { Bruttonationaleink.) }\end{array}$ & $\begin{array}{l}1.00 \\
(.00) \\
1.00 \\
(.00)\end{array}$ & $\begin{array}{l}1.00 \\
(.00) \\
1.00 \\
(.00)\end{array}$ & $\begin{array}{l}1.00 \\
(.00) \\
1.00 \\
(.00)\end{array}$ & $\begin{array}{l}1.00 \\
(.00) \\
1.00 \\
(.00)\end{array}$ & $\begin{array}{l}1.00 \\
(.00) \\
1.00 \\
(.00)\end{array}$ \\
\hline \multicolumn{6}{|l|}{ Regime } \\
\hline Demokratie & $\begin{array}{l}1.02 \\
(.02)\end{array}$ & $\begin{array}{l}1.05^{* *} \\
(.02)\end{array}$ & $\begin{array}{l}1.02 \\
(.02)\end{array}$ & $\begin{array}{l}1.02 \\
(.02)\end{array}$ & $\begin{array}{l}1.03 \\
(.02)\end{array}$ \\
\hline Föderales System & $\begin{array}{l}.50^{* *} \\
(.17)\end{array}$ & $\begin{array}{l}.51^{*} \\
(.18)\end{array}$ & $\begin{array}{l}.50^{* *} \\
(.17)\end{array}$ & $\begin{array}{l}.50^{* *} \\
(.17)\end{array}$ & $\begin{array}{l}.52^{*} \\
(.18)\end{array}$ \\
\hline (Ehemals) sozialistisch & $\begin{array}{l}2.10^{* *} \\
(.64)\end{array}$ & $\begin{array}{l}2.24 * * \\
(.71)\end{array}$ & $\begin{array}{l}2.16^{* * *} \\
(.68)\end{array}$ & $\begin{array}{l}2.08^{* *} \\
(.64)\end{array}$ & $\begin{array}{l}2.25^{* * *} \\
(.69)\end{array}$ \\
\hline \multicolumn{6}{|l|}{ Sozioökonomische Faktoren } \\
\hline $\begin{array}{l}\text { BIP pro Kopf (log) } \\
\text { Kinderarbeitsrate }\end{array}$ & $\begin{array}{l}.69 * * * \\
(.08) \\
1.00 \\
(.01)\end{array}$ & $\begin{array}{l}.85^{*} \\
.07) \\
1.00 \\
.01)\end{array}$ & $\begin{array}{l}.70^{* * *} \\
.08) \\
1.00 \\
(.01)\end{array}$ & $\begin{array}{l}.72^{* * *} \\
(.08) \\
1.00 \\
(.01)\end{array}$ & $\begin{array}{l}.92 \\
(.11) \\
1.00 \\
(.01)\end{array}$ \\
\hline \multicolumn{6}{|l|}{ Modellzusammenfassung } \\
\hline Anzahl der Länder & 106 & 106 & 106 & 106 & 106 \\
\hline $\begin{array}{l}\text { Anzahl der Beobachtungen } \\
\text { Länder/Jahr }\end{array}$ & 2458 & 2458 & 2458 & 2458 & 2458 \\
\hline Anzahl der Ratifizierungen & 91 & 91 & 91 & 91 & 91 \\
\hline LR chi" & -102.89 & -109.07 & -102.79 & -92.56 & -76.05 \\
\hline
\end{tabular}

Signifikanzen: ${ }^{*} \mathrm{p}<.1{ }^{* *} \mathrm{p}<.05{ }^{* * *} \mathrm{p}<.01$

Quelle: World Development Indicators, Union of International Associations, eigene Berechnung

Wie weltgesellschaftliche Erwägungen (Hypothese 1) prognostizieren, ist eine Ratifizierung wahrscheinlicher bei Ländern, die starke organisationale Bindungen zur Weltgesellschaft aufweisen. Modell 1 zeigt, dass die Mitgliedschaften in internationalen Regierungsorganisationen einen signifikanten positiven Effekt ausüben. Modell 2 zeigt, dass dies auch für Nichtregierungsorganisationen der Fall ist. In Modell 3 sind sowohl Regierungsorganisationen wie Nichtregierungsorganisationen enthalten. Wie 
in Hypothese 2 vermutet, verlieren die Regierungsorganisationen ihre Signifikanz, wenn NGOs in das Modell eingeführt werden. Obwohl die Interpretation der Ergebnisse mit Vorsicht erfolgen sollte, da eine beachtliche Kollinearität zwischen den beiden Variablen besteht, legen die Ergebnisse die größere Effektivität von NGOs nahe. Eine Etklärung wäre, dass, obwohl Regierungsorganisationen über mehr Ressourcen verfügen, auch NGOs Träger weltgesellschaftlicher Normen sind, denen zudem ein größeres Arsenal an Strategien zur Verfügung steht (Abu Sharkh 1998; Keck/Sikkink 1998). Während Regierungsorganisationen oft in einem diplomatischen Korsett gefangen sind, können NGOs die Aufmerksamkeit der Öffentlichkeit auf ein Thema lenken. Proteste sind gemäß der Literatur über soziale Bewegungen eine sehr effektive Strategie, um ein Thema auf die internationale Agenda zu bringen, wie die Aktivität von Menschenrechtsorganisationen wie Human Rights Watch oder Amnesty International zeigt. NGOs mobilisieren.

Modell 4 prüft den Effekt externer weltgesellschaftlicher "Schocks" durch die 1998 erlassene ILO-Deklaration zu Fundamentalen Arbeitsrechten. Die Wahrscheinlichkeit für Nicht-Ratifizierer, die Konvention im Jahr der Verabschiedung dieser Deklaration oder im darauf folgenden Jahr zu ratifizieren, ist beinahe fünf Mal so hoch wie in allen anderen Jahren. Die Bemühungen der ILO, dem Verbot von Kinderarbeit durch eine Weltkonferenz Aufmerksamkeit und zumindest symbolische Anerkennung durch Ratifizierung der Konventionen zu verschaffen, war somit erfolgreich.

Das letzte, fünfte Modell überprüft, ob die Ratifizierung anderer Kernarbeitskonventionen die Wahrscheinlichkeit erhöht, die Kinderarbeitskonvention zu ratifizieren, wie in Hypothese 4 vermutet. Die Ratifikation anderer Kernkonventionen erhöht die Wahrscheinlichkeit signifikant, auch die Kinderarbeitskonvention zu ratifizieren. Auch hier könnte die Einbindung in weltgesellschaftliche Arenen, hier durch vorhergehende Ratifizierungen, ausschlaggebend für Ratifizierungen sein. Für Länder, die bereits durch ein Netz von ratifizierten Konventionen mit internationalen Organisationen verbunden sind, mag es ein kleinerer Schritt sein, sich einer weiteren Konvention zu verpflichten. Diese Länder könnten ohnehin den fundamentalen Werten der ILO ideologisch verbunden sein.

Weiterführende Analysen legen nahe, dass die einflussreichste Kernkonvention die Nicht-Diskriminierungskonvention ist. Länder, die diese Konvention tatifiziert haben, ratifizieren die Kinderarbeitskonvention mit dreimal größerer Wahrscheinlichkeit. Die jeweils zwei Konventionen zu Kinderarbeit und Diskriminierung zu ratifizieren, könnte Teil eines strategischen Bemühens sein, arbeitsbezogene Menschenrechte aufrechtzuerhalten. Gemäß der Entwicklungsliteratur gehen Diskriminierung und Kinderarbeit oft miteinander einher. Die Barrieren, denen Frauen im Arbeitsmarkt ausgesetzt sind, machen es nötig, dass ihre Kinder arbeiten. Die Diskriminierung von Einwanderern und die damit einhergehende ökonomische Unsicherheit resultiert oft in der Verwendung der Arbeitskraft von Kindern als einer Art Absicherung.

Erwähnenswert ist auch, welche Variablen keine signifikanten Effekte auf die Ratifizierungswahrscheinlichkeit ausüben. Alle Variablen, die als Indikator für „Kultur" im weitesten Sinne gelten können, wie regionale Zugehörigkeit (Afrika, Asien etc.), ehemalige Kolonialmächte (Frankreich, Portugal etc.) oder die religiöse Ausrichtung der Bevölkerung (Islam, Protestantismus etc.), waren nicht signifikant. 
Dies galt bemerkenswerterweise auch für alle Indikatoren eines funktionierenden Schulsystems wie Einschulungsraten, Bildungsausgaben etc. Diese wurden als Kontrollvariablen getestet, da Kinder auch aufgrund mangelnder Alternativen, z.B. nicht vorhandener Schulsysteme, Arbeit aufnehmen könnten. Letztlich wurden auch Indikatoren für Diskriminierung wie das Verhältnis von Mädchen und Jungen im Schulsystem oder die weibliche Erwerbsbeteiligung am Arbeitsmarkt geprüft und für irrelevant befunden.

\section{Fazit}

Diese Resultate bestätigen für den Bereich von Kernarbeitskonventionen, was vorhergehende weltgesellschaftliche Untersuchungen für Frauenrechts- (Wotipka/Ramirez 2008) und Menschenrechtskonventionen (Wotipka/Tsutsui 2008) nachweisen konnten: Globale Diskurse und Initiativen, die von der internationalen Plattform durch weltweit agierende Organisationen in die einzelnen Länder getragen werden, prägen nationalstaatliches Ratifizierungsverhalten nachhaltig.

Weltgesellschaftliche Argumente untermauernd, zeigt diese Untersuchung, dass ökonomische Faktoren eine untergeordnete Rolle spielen. Arbeitsmarktstrukturen, die ein Land davon abhalten sollten, Vertragsversprechen einzugehen, die es nicht einlösen kann, sind insignifikant: Real existierende Kinderarbeit hält Länder nicht davon ab, sich, zumindest zeremoniell in der Form von Ratifizierung, gegen Kinderarbeit zu positionieren. Auch ökonomische Abhängigkeitsverhältnisse in der Form von Handel oder Entwicklungshilfe beeinflussen die Wahrscheinlichkeit, dass Länder die internationale Kinderarbeitskonvention ratifizieren, weder positiv noch negativ. Die Ratifizierung von Kinderarbeitsnormen, so legen diese Resultate nahe, wird nicht allein von funktionalen Arbeitsmarktfaktoren oder außenpolitischem Kalkül geprägt, wie es die Entwicklungsliteratur realpolitischer Prägung nahelegt.

Innenpolitische Faktoren sind jedoch für die Ratifizierung von Arbeitsmarktkonventionen relevanter als für die Unterzeichnung anderer Konventionen. HafnerBurton et al. (2008) argumentieren, dass innenpolitisches Kalkül keinen signifikanten Einfluss auf die Unterzeichnung von Menschenrechtskonventionen ausübt. Dies wird für den Bereich von Arbeitskonventionen nur bedingt bestätigt. Ideologische Erwägungen und Positionierung spielen für diese Art von Konventionen eine beachtliche Rolle. So sind (ehemals) sozialistische Staaten sehr viel geneigter, die Kinderarbeitskonvention zu ratifizieren. Der Staatsaufbau übt einen signifikanten Einfluss auf die Ratifizierungswahrscheinlichkeit aus. So sind föderale Staaten wesentlich weniger befähigt, Arbeitsmarktkonventionen zu ratifizieren, da der Zuständigkeitsbereich für Arbeitsmarktfragen nicht immer im bundesstaatlichen Hoheitsgebiet liegt.

Festzuhalten bleibt, dass das Ratifizierungsverhalten von Staaten stark von ideologischen Erwägungen (mit)bestimmt wird. Historisch spielen dabei sozialistische Vorstellungen eine Rolle. Aktuell prägen weltgesellschaftliche, organisatorische Einbindung und Ereignisse das Ratifizierungsverhalten hinsichtlich der Kinderarbeitskonvention.

Sozialpolitisch von Interesse ist diese Untersuchung, da sie suggerierte, dass der von den Vereinten Nationen und vielen NGOs verfolgte „Befähigungsansatz" inter- 
national eine Erfolg versprechende Strategie zu sein vermag. Vielfach als bloße „talk shops“ abgetan, scheinen UN-Organisationen und -Konferenzen sowie NGOAktivitäten dennoch eine beachtliche Wirkung hinsichtlich der Anerkennung weltgesellschaftlicher Normen durch Staaten zu zeitigen. Jedoch bleibt eine Ratifikation nur der erste Schritt auf dem Weg internationaler Normumsetzung. Eine weiterführende Frage wäre, unter welchen Umständen diese formalen Zusagen tatsächliche Arbeitsmarktgesetzesreform (Abu Sharkh 2004) und -veränderungen, also einen messbaren Abbau von Kinderarbeit, vorantreiben.?

\section{Literaturverzeichnis}

Abu Sharkh, Miriam (1998): „A Theoretical and Comparative Approach to the South African Women's Movement", in: Bernhard von der Haar (Hg.) (1997): Empirical Approaches to a Society in Negotiation. Berlin: Freie Universität, 47-99.

Abu Sharkh, Miriam (2000): Wachstum contra Gerecbtigkeit? Basispapier zu Sozialstandards in der Tecbnischen Zusammenarbeit. Eschborn: GTZ.

Abu Sharkh, Miriam (2002): History and Results of Labor Standard Initiatives. An Event History and Panel Analysis of the Ratification Patterns, and Effects, of the International Labour Organization's First Child Labour Convention. Institut für Soziologie, Freie Universität Berlin, Ph.D. dissertation.

Abu Sharkh, Miriam (2004): Fighting Child Labor: What Works and Why. A Panel Analysis of the Ratification Effects of the International Labour Organization's First Child Labor Convention and the Role of Transnational Advocacy Networks. "Transnational Risks and Civil Society", 10.-12. Januar 2004, Potsdam. Working paper.

Abu Sharkh, Miriam (2007): „Taking Labour Laws to the Dentist: Measuring Countries' Compliance with International Labour Non-discrimination Standards", in: David Kucera (Hg.): Measuring the Immeasurable: Qualitative Indicators of Labour Standards. Amsterdam: Springer, 21-31.

Abu Sharkh, Miriam (2009): Cross-coupling of national and international law regarding Labour Market Discrimination against Women 1958 to 2005. Stanford Center for Democracy, Development and the Rule of Law, Stanford University, Working paper No. 90.

Anker, Richard (1998): „Overview and Introduction“, in: Richard Anker; Sandhya Barge; S. Rajagopal; M.P. Joseph (Hg.): Economics of Child Labour in Hazardous Industries of India. New Delhi: Hindustan Publishing Corporation, 1-24.

Archavanikul, Kritaya (1998): Trafficking in Children for Labour Exploitation including Child Prostitution in the Mekong Sub-Region. Bangkok: ILO-IPEC.

Bequel, A.; Myers, W.E. (1995): First Things first in Child Labour. Eliminating Work Detrimental to Children. Geneva: ILO.

Berkovitch, Nitza (1999): From Motberbood to Citizenship: Women's Rights and International Organisations. Baltimore: John Hopkins University.

Boli, John (1987): Human Rights or State Expansion? Cross-National Definitions of Constitutional Rights, 1870-1970. London: Sage.

9 Siehe auch Hathaways Fragestellung (2002) in "Do Human Rights Treaties Make a Difference?". 
Boli, John; Meyer, John (1987): The Ideology of Childhood and the State: Rules Distinguishing Cbildren in National Constitutions. London: Sage.

Boli, John; Thomas, George (1999): „Introduction” and the „INGOs and the Organisation of World Culture", in: John Boli; George Thomas (Hg.): Constructing World Culture. Stanford: Stanford University, 1-49.

Bornschier, Volker (1996): „Weltgesellschaft“, in: Gerd Reinhold (Hg.): Soziologie-Lexikon. 3. Aufl. München: Oldenbourg, 717-720.

Bradley, Karen; Ramirez, Francisco O. (1996): „World Polity and Gender Parity: Women's Share of Higher Education, 1965-1985“, Research in Sociology of Education and Socialisation 11: 63-91.

Burton, John W. (1972): World Society. Cambridge: Cambridge University.

Canagarajah, Sudharshan; Coulombe, Harold (1997): Child labour and schooling in Gbana. Weltbank: Research Working Paper.

Charnovitz, Steve (1994): The World Trade Organisation and Social Issues. Presentation made at the Conference on „The Future of the Trading System". University of Ottawa.

Cleves, Mario; Gould, William; Gutierrez, Roberto; Marchenko, Yulia (2008): An Introduction to Survival Analysis. College Station: STATA.

Compa, Lance (1993): „Labour Rights and Labour Standards in International Trade”, Law and Poligy in International Business 25: 165-191.

DiMaggio, Paul; Powell, Walter (1991): „Introduction”, in: Walter Powell; Paul DiMaggio (Hg.): The New Institutionalism in Organisational Analysis. Chicago: Chicago University Press, 1-40.

Döhring, Karl (1999): Völkerrecbt. Fin I ebrbuch. Heidelberg: Müller.

Fallon, Peter; Zafiris, Tzannatos (1998): Child Labour: Issues and Directions for the World Bank. Washington, D.C., World Bank, Research Paper.

Finnemore, Martha (1996): „Norms, Culture and World Politics: Insights from Sociology's Institutionalism", International Organisation 50 (2): 325-47.

Forschungsgruppe Weltgesellschaft (1996): „Weltgesellschaft: Identifizierung eines Phantoms", Politiscbe Vierteljabresschrift 37 (1): 5-26.

Frank, Volker (1999): „Die Durchsetzung internationaler Standards - Erfolgsbedingungen von

Sozialklauseln", Peripberie. Zeitschrift für Politik und Ökonomie in der Dritten Welt 19 (75): 66-82.

Frey, Barbara (1997): „The Legal and Ethical Responsibilities of Transnational Corporations in the Protection of International Human Rights", Minnesota Journal on Global Trade 6 (105): 153-188.

Gamson, William (1997): „The Success of the Unruly”, in: Doug McAdam; David Snow (Hg): Social Movements: Readings on their Emergence, Mobilization, and Dynamics. Arizona: University of Arizona, 357-365.

Grattet, Ryken (1998): Substantiating a Worker's Right to Compensation. New York u.a.: Garland Publishing.

Greven, Thomas; Scherrer, Christoph (1998): „Die soziale Flankierung des Weltmarkts - Eine Einführung“, in: Christoph Scherrer; Thomas Greven; Volker Frank (Hg.): Sozialklauseln. Münster: Westfälisches Dampfboot, 12-32.

Grootaert, Christiaan (1998): Child Labour in Cote d'Ivoire: Incidents and Determinants, Washington, D.C., World Bank, Research Paper.

Großmann, Harald; Koopmann, Georg (1994): „Sozialstandards für den internationalen Handel?“" Wirtschaftsdienst 11: 585-591. 
Grote, Ulrike; Basu, Arnab; Reinhold, Diana (1998): "Child Labour and International Policy Debate - The Education/Child Labour Trade-Off and the Consequences of Sanctions", Military Sociology 18: 1-23.

Hafner-Burton, Emilie; Tsutsui, Kiyoteru; Meyer, John (2008): „International Human Rights Law and the Politics of Legitimacy: Repressive States and Human Rights Treaties", in: International Sociology 23 (1): 115-141.

Hathaway, Oona A. (2002): „Do Human Rights Treaties Make a Difference?”, The Yale Law Journal 111: 1935-2042.

Hannan, Michael T.; Tuma, Nancy Brandon (1979): „Methods for Temporal Analysis”, Annual Review of Sociology 5: 303-328.

Hironaka, Ann (1997): Boundaries of War: Historical Changes in Types of War, 1816-1980. Stanford: Stanford University.

ILO (International Labour Office) (1997): Standard Setting and Globalisation. Genf: ILO.

Jennings, Norman (1999): Child Labour in Small-Scale Mining: Examples from Niger, Peru and Pbilippines. Genf: ILO.

Keck, Margaret E.; Kathryn Sikkink (1998): Activists Beyond Borders: Advocacy Networks in International Politics. London: Cornell University Press.

Kellerson, Hilary (1998): „The ILO Declaration of 1998 on Fundamental Rights and Principles: A Challenge for the Future", International Labour Review 137 (22): 223-227.

Krasner, Stephen (2000): „International Law and International Relations: Together, Apart, Together?", Chicago Journal of International Law 1 (1): 93-99.

Kulessa, Margareta (1995): Handelspolitische Sozialstandards zum Woble der ,Dritten Welt'? Duisburg: Gerhard-Mercator-Universität.

Langhammer, Rolf (1999): The WTO and the Millennium Round. Between Standstill and Leapfrog. Kiel: Institut für Weltwirtschaft.

Leisering, Lutz/Tao, Liu (2010): „Globale Wissensdiffusion in der Sozialpolitik. Die Einführung einer Arbeitsunfallversicherung in der Volksrepublik China", Zeitschrift für Sozialreform 56 (2): 173-205.

Leisink, Peter (1999): „Introduction”, in: Peter Leisink (Hg.): Globalisation and Labour Relations. Cheltenham: Edward Elgar, 1-35.

Levison, Deborah; Anker, Richard; Ashraf, Shahid; Barge, Sandhya (1996): Is Child Labour Really Necessary in Indias Carpet Industry? Genf: ILO.

Liemt, Gijsbert van (1989): „Minimum Labour Standards and International Trade: Would a Social Clause Work?”, International Labour Review 128 (4): 433-448.

Liese, Andrea (2005): „Capacity Building' als Strategie zur Förderung der Regeleinhaltung. Erfahrungen der ILO bei der Abschaffung von Kinderarbeit", in: Eva SenghaasKnobloch $(\mathrm{Hg}$.): Weltweit geltende Arbeitsstandards trotz Globalisierung. Analysen, Diagnosen und Einblicke. Münster: LIT Verlag, 63-79.

Malanowski, Norbert (1997): Social and Environmental Standards in International Trade Agreements: Links, Implementations and Prospects. Münster: Westfälisches Dampfboot.

Maskus, Keith (1997): Should Core Labour Standards Be Imposed Through International Trade Policy? Washington, D.C., World Bank, Policy Research Working Paper Nr. 1817.

McAdam, Doug; McCarthy, John; 'Lald, Mayer N. (1996): „Introduction: Opportunities, Mobilizing Structures, and Framing Processes", in: Doug McAdam; John D. McCarthy; Mayer N. Zald (Hg.): Comparative Perspectives on Social Movements. Cambridge: Cambridge University, 1-20. 
McNeely, Connie L. (1998): Constituting Citizens. Rights and Rules. New York u.a.: Garland Publishing.

Meyer, John W. (1987): The World Polity and the Autbority of the Nation-State. London: Sage.

Meyer, John W.; Boli, John; Thomas, George; Ramirez, Francisco (1997): „World Society and the Nation State", American Journal of Sociology 103 (1): 144-181.

Meyer, John W.; Ramirez, Francisco O.; Soysal, Yasemin Nuhoglu (1992): „World Expansion of Mass Education, 1870-1980", Sociology of Education 65: 128-149.

Meyer, John W.; Rowan, Brian (1977): „Institutionalized Organisations: Formal Structures as Myth and Ceremony", American Joumal of Sociology 83 (2): 340-363.

Nuschler, Franz (1996): Lem- und Arbeitsbuch Entwicklungspolitik. Bonn: Dietz.

OECD (Organisation for Economic Co-Operation and Development) (1995): Trade and Labour Standards: A Review of the Issue. Paris: OECD.

Orloff, Ann Shola (1998): Gendering the Analysis of Welfare States. With Examples from Australia and the United States. New York u.a.: Garland Publishing.

Perez-Lopez, Jorge (1987): Conditioning Trade on Foreign Labour Law: The U.S. Approach. Paper presented at a panel on "Worker's Rights and International Trade" at the 81 th Meeting of the American Society of International Law, Boston, Massachusetts.

Ramirez, Francisco O. (2000): „Progress, Justice, and Gender Equity: World Models and Cross-National Trends", in: Walter Powell; Dan Jones (Hg.): Bending the Bars of the Iron Cage: Institutional Dynamics and Processes. Chicago: Chicago University, 40-53.

Ramirez, Francisco O.; Meyer, John W. (1998): „Dynamics of Citizenship Development and the Political Incorporation of Women", in: Connic L. McNeely (Hg.): Public Rights, Public Rules: Constituting Citizens in the World Polity and National Policy. New York u.a.: Garland Publishing, 59-80.

Ramirez, Francisco O.; Soysal, Yasemin; Shanahan, Suzanne (1997): „The Changing Logic of Political Citizenship: Cross-National Acquision of Women's Suffrage Rights, 18901990", American Sociological Review 62 (5): 735-745. Ravallion, Martin; Wodon, Quentin (1999): Does Child Labour Displace Schooling? Evidence on behavioral responses to an enrollment subsidy. Washington, D.C.: World Bank.

Risse, Thomas; Sikkink, Kathryn (1999): „The socialization of international human rights norms into domestic practises: introduction", in: Thomas Risse; Stephen C. Ropp; Kathryn Sikkink ( $\mathrm{Hg}$.): The Power of Human Rights. International Norms and Domestic Change. Cambridge: Cambridge University Press, 1-38.

Robertson, Roland (1992): „Globality, Global Culture, and Images of World Order”, in: Hans Haferkamp; Neil J. Smelser (Hg.): Social Change and Modernity. Berkeley: University of California, 395-411.

Sapir, André (1995): „The Interaction between Labour Standards and International Trade Policy", The World Economy 18 (6): 791-803.

Schäfer, Dieter (1993): „Sozialpolitik“, in: Deutscher Verein für öffentliche und private Fürsorge (Hg.): Facblexikon der sozialen Arbeit. Frankfurt am Main: Nomos.

Scherrer, Christoph (1998): „Die wissenschaftliche Diskussion“, in: Christoph Scherrer; Thomas Greven; Volker Frank (Hg.): Sozialklauseln. Münster: Westfälisches Dampfboot, 34-110.

Senghaas-Knobloch, Eva (1999): „Die Internationale Arbeitsorganisation vor neuen Herausforderungen der Globalisierung“, Peripherie. Zeitschrift für Politik und Ökonomie in der Dritten Welt 19 (75): 26-43. 
Senghaas-Knobloch, Eva (2005): „Geltung und Wirksamkeit internationaler Regulierungen durch die Internationale Arbeitsorganisation (ILO) in Zeiten der Globalisierung - Eckpunkte und Diskussionslinien", in: Eva Senghaas-Knobloch ( $\mathrm{Hg}$.): Weltweit geltende Arbeitsstandards trotz Globalisierung. Analysen, Diagnosen und Einblicke. Münster: LIT Verlag, 5-33.

Simmons, Beth; Hopkins, Daniel (2005): „The Constraining Power of International Treaties“, American Political Science Review 99 (4): 623-631.

Tallberg, Jonas (2002): „Paths to Compliance: Enforcement, Management, and the European Union", Intermational Organization 56 (3): 609-643.

Thomas, George M.; Lauderdale, Pat (1988): „State Authority and National Welfare Programs in the World System Context", Sociological Forum 3 (3): 383-399.

Tronto, Joan (1991): „Changing Goals and Changing Strategies: Varieties of Women's Political Activism", Feminist Studies 17 (1): 85-104.

Tsutsui, Kiyoteru (1998): Global Human Rights and Etbnic Minority Movements: 1945-1995. Stanford: Stanford University.

Wet, Erika de (1998): „Labour Standards in the Globalized Economy: The Inclusion of a Social Clause in the General Agreement on Tariff and Trade/World Trade Organisation", Human Rights Quarterly 17 (3): 143-462.

Windfuhr, Michael (1999): „Durchsetzung sozialer Menschenrechte. Problemdimensionen und Vergleich der Reichweite zivilgesellschaftlicher Kampagnen und Initiativen“, Peripherie. Zeitschrift für Politik und Ölonomie in der Dritten Welt 19 (75): 23-40.

Wobbe, Theresa (2000): Weltgesellscbaft. Bielefeld: transcript.

World Bank (2000): World Development Report 2000. Washington, D.C.: World Bank.

Wotipka, Christine Min; Ramirez, Francisco O. (2008): „World Society and Human Rights: An Event History Analysis of the Convention on the Elimination of All Forms of Discrimination against Women", in: Beth A. Simmons; Frank Dobbin; Geoffrey Garrett (Hg.): The Global Diffusion of Markets and Democracy. Cambridge: Cambridge University Press, 303-343.

Wotipka, Christine Min; Tsutsui, Kiyoteru (2008): „Global Human Rights and State Sovereignty: Nation-States' Ratifications of International Human Rights Treaties, 19652001", Sociological Forum 23 (4): 724-754.

Zeeb, Matthias (1994): „Gegen einseitige Verbote“, EPD-Entwicklungspolitik 7/8: 29-30.

Zürn, Michael (1997): „Positives Regieren' jenseits des Nationalstaats. Zur Implementation internationaler Umweltregime”, Zeitschrift für Internationale Besiehungen 4 (1): 41-68. 


\section{Kontakt}

Dr. Miriam Abu Sharkh

Standford Center for International Development

366 Calvez St.

Stanford University

Stanford, CA 94305

E-Mail: mabu@stanford.edu

http://cddrl.stanford.edu/people/miriamabusharkh/

Miriam Abu Sharkh ist gegenwärtig Visiting Associate Professor am Stanford Center for International Development und Visiting Scholar am Center for Democracy, Development and the Rule of Law (ebenfalls Universität Standford). Zuvor arbeitet Miriam Abu Shark u.a. bei der ILO und war für die Gesellschaft für Technische Zusammenarbeit (GTZ) als Beraterin tätig. Ihre Forschungsschwerpunkte liegen im Bereich der Menschentechte, Kernarbeitsrechte, Kinderarbeit, Geschlechterdiskriminierung und Wohlfahrtsregime. 
Brought to you by | London School of Economics and Political Science

Authenticated

Download Date | 7/10/17 6:02 PM 